\title{
Significant carrier concentration changes in native electrodeposited ZnO
}

\author{
Shawn Chatman, Lisa Emberley, and Kristin M. Poduska* \\ Department of Physics and Physical Oceanography, Memorial University of Newfoundland, \\ St. John's, NL, A1B 3X7, Canada \\ E-mail: kris@mun.ca,Phone:(709)-737-8890,Fax:(709)737-8739
}


Shawn Chatman, Lisa Emberley, and Kristin M. PoduskaSignificant carrier concentration changes ...

\begin{abstract}
We show that unintentional hydrogen doping of $\mathrm{ZnO}$ during the electrodeposition process can impact the material's carrier concentration as significantly as others have reported for intentional extrinsic doping. Mott-Schottky analyses on the natively $n$-type electrodeposits show a decrease in carrier concentrations from $10^{21}$ to $10^{18} \mathrm{~cm}^{-3}$ with increasing overpotential. A strong link exists between larger optical band gaps (determined from diffuse reflectance spectroscopy) and higher carrier concentrations, which suggests that hydrogen-based doping underlies the $n$-type conductivity (Moss-Burstein effect). We propose that kinetic defects introduced during growth at larger overpotentials compete with hydrogen doping, thereby leading to lower net carrier concentrations. This has important implications for using deposition potential to tune other electrodeposit properties such as growth rate and morphology.
\end{abstract}

Keywords: $\mathrm{ZnO}$, carrier concentration, hydrogen doping, band gap, electrodeposition, X-ray diffraction 
Shawn Chatman, Lisa Emberley, and Kristin M. PoduskaSignificant carrier concentration changes ...

\section{Introduction}

Transparent semiconducting materials are the cornerstone of many optoelectronic devices, including those used in the photovoltaic industry. ${ }^{1}$ In these applications, reliable control of carrier concentration is essential to optimize both optical transparency and electrical conductivity. ${ }^{2}$ Electrochemical deposition of transparent semiconductors is gaining acceptance as a viable means of producing films of transparent semiconducting metal oxides such as $\mathrm{ZnO}$ and $\mathrm{TiO}_{2} \cdot{ }^{3}$ However, there is still much to be understood about how to control carrier concentrations in these electrodeposited materials. Here, we show that the carrier concentration of native electrodeposited $\mathrm{ZnO}$ can be modified over several orders of magnitude simply by changing the potential applied during deposition. Our results show an approach to $n$-doping that yields a similar range of carrier concentrations without introducing intentional dopants. The implications are that using potential to control other features of the electrodeposit, such as growth rate or morphology, may simultaneously affect the deposit's electronic properties.

$\mathrm{ZnO}$ is a well-studied wide band gap material that is $n$-type in its native form. ${ }^{2,4}$ Considerable scientific effort has been devoted to understanding how and why hydrogen is the cause of this $n$-type doping. ${ }^{5,6}$ Hydrogen is expected to be an amphoteric dopant in most materials by compensating existing defects. In $\mathrm{ZnO}$, however, electronically active interstitial $\mathrm{H}^{+}$serves as a donor ${ }^{7}$ and increases the band gap energy when doping levels are high enough to lead to degenerate semi-

conducting behavior. This Moss-Burstein effect ${ }^{8}$ of larger band gaps with higher doping levels is a trend that opposes the usual response of decreasing band gap with an increase in extrinsic dopants (as has been observed in boron-doped $\mathrm{ZnO}^{9}$ and more recently with $\mathrm{Cl}^{-}$in $\mathrm{ZnO}$ electrodeposits $^{10}$ ).

This work, based on Mott-Schottky analyses, shows that significant changes in carrier concentration can be induced in native $\mathrm{ZnO}$ electrodeposits without introducing intentional dopants. Since the hydrogen evolution reaction (HER) proceeds concurrently with $\mathrm{ZnO}$ electrosynthesis, there is an obvious presence of hydrogen. However, there are no existing studies that address its effect on carrier concentration. 
Shawn Chatman, Lisa Emberley, and Kristin M. PoduskaSignificant carrier concentration changes ...

\section{Experimental methods}

\section{ZnO electrosynthesis}

$\mathrm{ZnO}$ thin films were deposited from aqueous electrolytes using a precipitation/reduction reaction process reported by Izaki et al. ${ }^{8}$ and modified by our research group..$^{4,5}$

$$
\begin{gathered}
\mathrm{NO}_{3}^{-}+\mathrm{H}_{2} \mathrm{O}+2 \mathrm{e}^{-} \rightarrow \mathrm{NO}_{2}^{-}+2 \mathrm{OH}^{-} \\
\mathrm{Zn}^{2+}+2 \mathrm{OH}^{-} \rightarrow \mathrm{Zn}(\mathrm{OH})_{2} \rightarrow \mathrm{ZnO}+\mathrm{H}_{2} \mathrm{O}
\end{gathered}
$$

During this reaction, nitrate ions are reduced to nitrite ions in the presence of $\mathrm{Zn}^{2+}$ adsorbed on the

surface of the working electrode. ${ }^{14}$ Consequently, excess hydroxide ions are produced, increasing the local $p \mathrm{H}$. This $p \mathrm{H}$ increase facilitates the formation of $\mathrm{Zn}(\mathrm{OH})_{2}$ on the working electrode, which spontaneously decomposes to $\mathrm{ZnO}$ at temperatures above $50^{\circ} \mathrm{C} .{ }^{14,15}$

$\mathrm{ZnO}$ samples were synthesized from $0.01 \mathrm{M} \mathrm{Zn}\left(\mathrm{NO}_{3}\right)_{2}$ (ACS reagent grade, SCP Science in 18.2 $\mathrm{M} \Omega \cdot \mathrm{cm}$ water, Barnstead Nanopure). Electrolyte $p \mathrm{H}$ was controlled by the addition of $\mathrm{HCl}$ $(p \mathrm{H}=4.0-5.5)$ or $\mathrm{NaOH}(p \mathrm{H}=6.0-7.5)$. Deposition potentials, ranging from $-1.3 \mathrm{~V}$ to $-0.85 \mathrm{~V}$, are all reported relative to a $\mathrm{Ag} / \mathrm{AgCl}$ reference. Substrates consisted of mechanically polished stainless steel (316 stainless steel or A286 steel alloy) that were ultrasonically cleaned prior to use.

\section{Structural, morphological, and optical characterization}

X-ray diffraction (XRD) data were collected using a Debye-Scherrer powder diffractometer (Rigaku D/MAX $2200 \mathrm{PC}$ ) in the $\theta-\theta$ geometry using the $\mathrm{Cu} K \alpha$ radiation. Diffraction data were collected over $20-80^{\circ} 2 \theta$, with a $0.03^{\circ} 2 \theta$ step size at $6^{\circ} / \mathrm{min}$.

Scanning electron microscopy (SEM) was performed with a Hitachi S570 scanning electron microscope and digital imaging collector. Deposit thicknesses were extracted from contact-mode atomic force microscopy (AFM) data taken with an Asylum Research MFP-3D system using a Si 
Shawn Chatman, Lisa Emberley, and Kristin M. PoduskaSignificant carrier concentration changes ...

tip ( $\mu$ Masch CSC37/Cr-Au with spring constant 0.1-0.4 N/m).

Diffuse reflectance spectroscopy data were collected using a Ocean Optics USB2000 spectrometer. The diffuse reflectance setup uses a UV/Visible light source surrounded by collectors incident upon the sample at $45^{\circ} . \mathrm{ZnO}$ band gaps were calculated from reflectance data by differentiating the reflectance intensity at the optical edge with respect to energy and taking the peak position of the first derivative, since other studies have shown that band gap values obtained with this method are closer to the true values obtained from transmission measurements. ${ }^{7}$

\section{Carrier concentration measurements}

Mott-Schottky (MS) analysis, based on the concept of impedance spectroscopy, utilizes the relationship between capacitance and applied potential to extract information about a material's carrier concentration. This technique has been applied to nano-structured ${ }^{9,10}$ as well as $\mathrm{Cl}$-doped ${ }^{10} \mathrm{ZnO}$.

MS analysis assesses carrier concentrations near a rectifying (non-ohmic, Schottky) junction at the surface of the semiconductor; in our experiments, this was achieved at the semiconductor/electrolyte interface. For Schottky junctions, theory dictates that the reciprocal of the junction capacitance squared will vary linearly with the applied reverse bias, as shown here (and derived in the Supporting Information):

$$
\left(\frac{1}{C}\right)^{2}=\frac{2\left(V_{b i}\right)+V_{R}}{e \varepsilon_{s} a^{2} N_{d}} .
$$

Here, $C$ is the junction capacitance, $V_{R}$ is the applied reverse bias voltage, $V_{b i}$ is the built-in potential, $a$ is the surface area of the junction, $e$ is the elemental charge, $N_{d}$ is the carrier concentration, and $\varepsilon_{s}$ is the semiconductor's dielectric constant, which we take to be 8.0 for bulk $\mathrm{ZnO} .{ }^{9}$ In reality, extra terms must be added to account for additional capacitive effects due to classic dielectric capacitance, $C_{d}$, as well as the "double-layer" capacitance, $C_{d l}$ (due to an accumulation of ions at the surface of the working electrode), and capacitance due to fractional coverage or a conducting substrate $(f)$ and increased surface area fraction due to roughness ( $g$, where for example $g=1.5$ 
Shawn Chatman, Lisa Emberley, and Kristin M. PoduskaSignificant carrier concentration changes ...

would indicate a $50 \%$ increase in surface area due to roughness): ${ }^{19}$

$$
\left(\frac{1}{C}\right)^{2}=\left(\frac{2\left(V_{b i}\right)+V_{R}}{e \varepsilon_{s} a^{2} N_{d}}\right)+(1-f) \frac{1}{C_{d l}^{2}}+f\left(\frac{1}{C_{d}}+\frac{1}{g C_{d l}}\right)^{2}
$$

Since these additional capacitance terms change the intercept - but not the slope - of Eq. 3, it is not necessary to quantify all auxiliary capacitances in order to determine the carrier concentration.

Accounting for the true surface area, $a$, of the $\mathrm{ZnO}$ in contact with the electrolyte requires prudent approximations. We assume that the planar surface area (as used in other investigations ${ }^{1}$ of $\mathrm{ZnO}$ electrodeposits) is an underestimation of the true surface area, suggesting the carrier concentrations determined from our MS data are upper limits. Therefore, we focus on trends and order of magnitude variations in carrier concentrations, rather than on specific values. A more comprehensive discussion of roughness effects on carrier concentration calculations is included in the Supporting Information.

MS analyses were performed in electrolyte by superimposing an alternating current at 10-15 $\mathrm{kHz}$ with a peak-to-peak voltage of $20 \mathrm{mV}$ over a stepped potential ranging from $-1 \mathrm{~V}$ to +1.3 $\mathrm{V}$ vs. $\mathrm{Ag} / \mathrm{AgCl}$ and measuring the resulting impedance. Capacitance values were extracted from impedance data using the relation:

$$
C=\frac{1}{j \omega Z}
$$

MS experiments were controlled by, and data were collected with, the PowerSUITE (EG\&G Princeton Applied Research (PAR)) impedance spectroscopy software package interfaced with a PAR 273A potentiostat and Signal Recovery 5210 lock-in amplifier. Several supporting electrolytes were tested for their suitability for these MS experiments. The native electrolyte $(p \mathrm{H}$ adjusted $\left.0.01 \mathrm{M} \mathrm{Zn}\left(\mathrm{NO}_{3}\right)_{2}\right)$, as used by Windisch et al., ${ }^{9}$ contributes capacitance fluctuations due to both the nitrate reduction reaction and $\mathrm{Zn}^{2+}$ adsorbtion. ${ }^{20} \mathrm{~A}$ better alternative was a modified phosphate buffer, similar to one reported recently, ${ }^{10}$ consisting of $0.063 \mathrm{M} \mathrm{K}_{2} \mathrm{PO}_{4}+.036 \mathrm{M}$ $\mathrm{NaOH}$, which has a $p \mathrm{H}$ of 7 . All MS data shown here were collected using this buffered phosphate 
Shawn Chatman, Lisa Emberley, and Kristin M. PoduskaSignificant carrier concentration changes ...

electrolyte.

\section{Results}

The electrodeposition process yields $\mathrm{ZnO}$ thin films whose lattice constants were refined to $3.246 \pm 0.001$ $\AA$ and $c=5.205 \pm 0.002 \AA$, which is in excellent agreement with JCPDS \#36-1451 for ZnO (3.250 $\AA$ and $5.207 \AA$ A). ${ }^{21}$ The refined lattice constants were consistent for samples deposited over a range of $p \mathrm{H}$ values (5.5-7.5) and deposition potentials $(-1.3 \mathrm{~V}$ to $-0.85 \mathrm{~V})$, consistent with previous results from our group over a more acidic $p \mathrm{H}$ range (4-5.5). ${ }^{5}$ Qualitative comparisons of relative peak heights (Figure 1) also confirmed that, while our samples are polycrystalline, there is a preferred (002) orientation in the deposit, which is typical for electrodeposited $\mathrm{ZnO} .^{4,15,22}$ This (002) texture is moderately accentuated for samples deposited at $-1.1 \mathrm{~V}(p \mathrm{H} 6.5)$, coinciding with a morphological change from hexagonal crystallites to rice-like structures, as shown in Figure 2, and reported previously. ${ }^{4}$

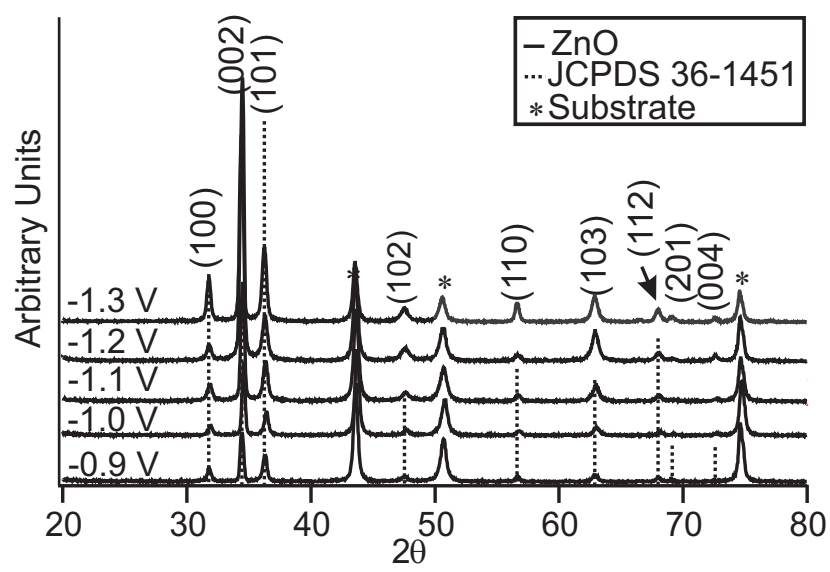

Figure 1: Representative indexed $\mathrm{XRD}$ patterns from electrodeposited $\mathrm{ZnO}$ prepared at $p \mathrm{H} 6.5$ with deposition potentials ranging from $-1.3 \mathrm{~V}$ to $-0.9 \mathrm{~V} v s$. $\mathrm{Ag} / \mathrm{AgCl}$. Refined lattice constants for electrodeposits deposited over the complete range of deposition conditions compared well with the accepted values $(a=3.250 \AA$ and $c=5.207 \AA$, JCPDS \#36-1451). Plots are offset along the intensity axis for clarity.

Despite the fact that XRD shows no obvious structural differences among samples prepared over this potential $(-0.9 \mathrm{~V}$ to $-1.3 \mathrm{~V})$ and $p \mathrm{H}$ range $(p \mathrm{H} 4-7)$, we find drastically different carrier 


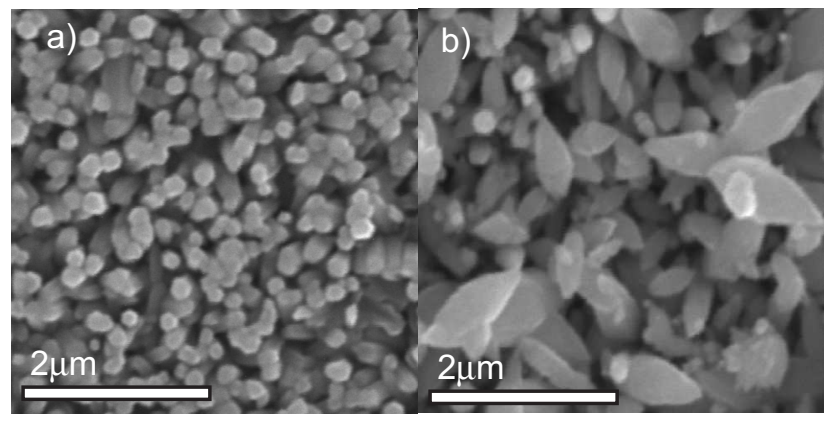

Figure 2: Representative SEM micrographs of $\mathrm{ZnO}$ samples deposited at (a) -0.9 V in $p \mathrm{H} 6.5$ electrolyte and (b) $-1.3 \mathrm{~V}$ in $p \mathrm{H} 5$ electrolyte. The hexagonal columns in (a) are typical of deposits from neutral $p \mathrm{H}$ electrolytes and more positive deposition potentials, while the rice-like morphologies are observed at more negative potentials and more acidic $p \mathrm{H}$ values.

concentrations based on MS analyses. Representative MS plots for samples deposited at low (-1.1 V) and high (-0.9 V) applied potentials (from $p \mathrm{H} 6.5$ electrolyte) are shown in Figure 3, corresponding to carrier concentrations of $8.7 \times 10^{20} \mathrm{~cm}^{-3}$ and $2.3 \times 10^{19} \mathrm{~cm}^{-3}$, respectively. Over a range of applied deposition potentials (all in $p \mathrm{H} 6.5$ electrolyte), there is a clear trend of decreasing carrier concentration with more negative deposition potentials, as shown in Figure 4a. Comparing data from electrodeposits prepared at different $p \mathrm{H}$ values, Figure $4 \mathrm{~b}$ shows carrier concentration versus the standard potential of the hydrogen evolution reaction (HER). Since the potential at which hydrogen evolves in solution is dependent upon $p \mathrm{H}$, this effectively couples potential and $p \mathrm{H}$, allowing a more direct comparison of carrier concentration change with deposition conditions. Both Figure 4a and Figure 4b show that carrier concentration decreases with increasing overpotential (with respect to HER). This relationship was established based on measurements of dozens of samples. Carrier concentrations were calculated from the linear section of the MS graphs, to eliminate the possibility of including data collected under complete depletion. We confirmed that deposit thicknesses $(0.5-3.0 \mu \mathrm{m})$ were all substantially larger than depletion layer thicknesses, even for the lowest carrier concentrations $\left(80 \mathrm{~nm}\right.$ at $10^{17} \mathrm{~cm}^{-3}$ to $8 \AA$ at $\left.10^{21} \mathrm{~cm}^{-3}\right)$.

Since carrier concentration changes can influence optical as well as electronic properties, we also compared trends in optical absorption edges, obtained from diffuse reflectance spectra, with electrodeposition conditions. Samples deposited at more positive potentials exhibited larger band 
Shawn Chatman, Lisa Emberley, and Kristin M. PoduskaSignificant carrier concentration changes ...

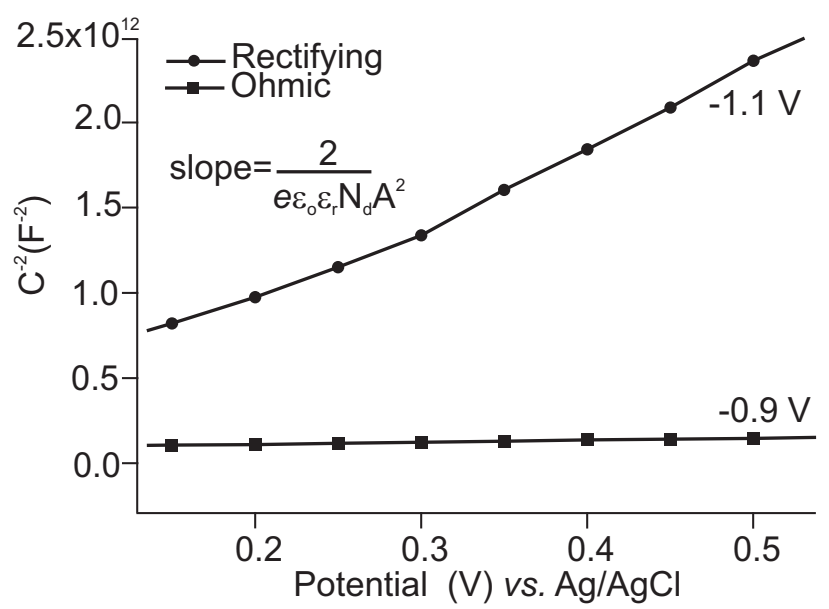

Figure 3: Representative $\mathrm{C}^{-2}$ vs. potential plots obtained from solution Mott-Schottky analyses performed on electrodeposited $\mathrm{ZnO}$ samples in $p \mathrm{H} 7$ phosphate buffer. The uncertainties associated with individual data points are smaller than the uncertainty calculated from the subsequent linear regression analysis.

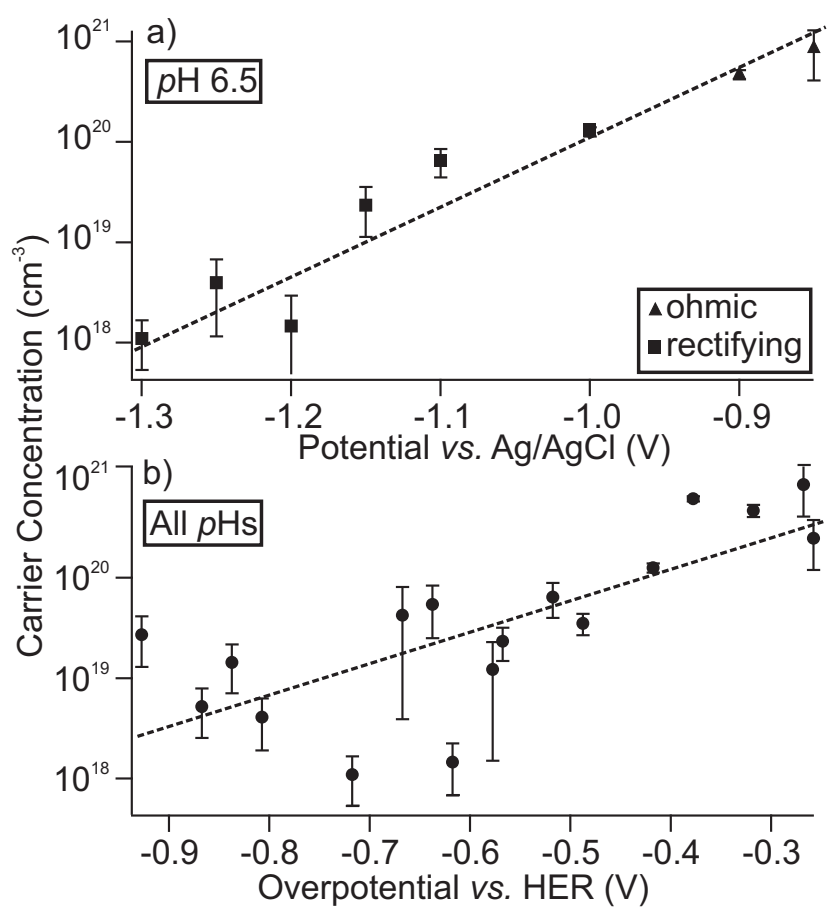

Figure 4: Carrier concentrations determined by solution Mott-Schottky analyses indicate a monotonic increase in carrier concentration with more positive deposition potentials. This figure shows plots of $\log$ carrier concentration versus overpotential (a) at $p \mathrm{H} 6.5$ and (b) for all $p \mathrm{H}$ conditions. 
Shawn Chatman, Lisa Emberley, and Kristin M. PoduskaSignificant carrier concentration changes ...

gaps than samples deposited at more negative deposition potentials, as can be seen in Figure 5a (samples synthesized at $p \mathrm{H}$ 6.5) and Figure 5b (all samples). Optical band gaps ranged between $3.1 \mathrm{eV}$ and $3.4 \mathrm{eV}$, which is in agreement with values reported for the band gap of $\mathrm{ZnO} .^{2,7,9,10} \mathrm{We}$ do not observe changes in band gap with changes in sample thickness between 0.5 and $3.0 \mu \mathrm{m}$. Although others ${ }^{7}$ have reported thickness dependent band gaps, their electrodeposits also show thickness-dependent changes in texture, morphology, and unit cell size that we do not observe in our electrodeposits. Additional discussions of the role of deposit thickness on optical band gap values are included in the Supporting Information.

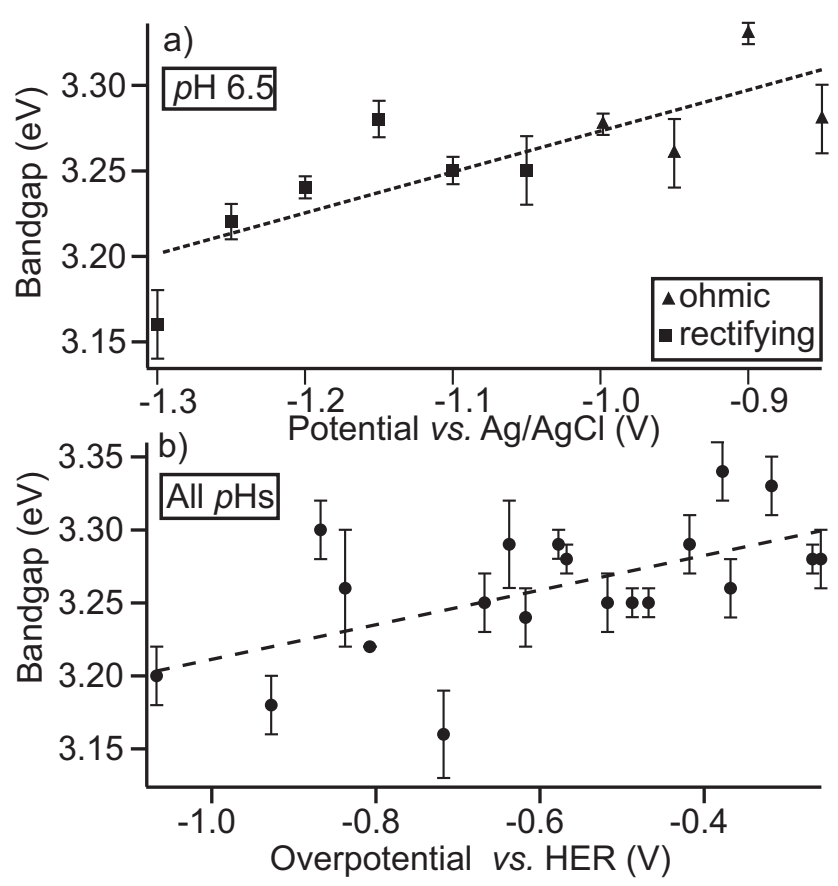

Figure 5: UV/Visible diffuse reflectance analysis was used to collect sample band gap values. Our data indicates a monotonic increase in band gap with more positive deposition potentials, (a) for samples deposited $p \mathrm{H} 6.5$ (plotted against a $\mathrm{Ag} / \mathrm{AgCl}$ reference) and (b) for all samples (the water electrolysis standard potential).

We note that built-in potentials could not be determined from our MS plots due to a sampledependent constant capacitance term. This may be due to variations in the electrodeposit coverage (parameter $f$ in Eq. 4), since earlier studies ${ }^{5}$ have shown that small pinholes can occur in our electrodeposits. Fabregat-Santiago et al. have also shown that sample-dependent capacitance can occur when there are sample/electrolyte interactions. ${ }^{19}$ 
Shawn Chatman, Lisa Emberley, and Kristin M. PoduskaSignificant carrier concentration changes ...

\section{Discussion}

Over the entire range of deposition potentials (-1.3 to $-0.85 \mathrm{~V})$ and $p \mathrm{H}$ values (4.0 to 7.5) studied, $\mathrm{ZnO}$ carrier concentrations ranged between $10^{17} \mathrm{~cm}^{-3}$ and $10^{20} \mathrm{~cm}^{-3}$. This range is in agreement with reported carrier concentrations for $\mathrm{ZnO},{ }^{9,10}$ including $\mathrm{ZnO}$ electrodeposited from nitratebased electrolytes. ${ }^{9,10}$

Our results indicate a higher $n$-type carrier concentration in $\mathrm{ZnO}$ that is electrodeposited at more positive deposition potentials. While some electrolytes, such as those containing $\mathrm{Cl}^{-}$or borane, ${ }^{9,10}$ offer a clear opportunity for extrinsic doping, the same cannot be said for our nitratebased electrolyte. Instead, it is much more likely that hydrogen is incorporated as an $n$-type dopant. We support this claim by correlating the trends in carrier concentration and optical band gap with respect to deposition potential.

Figure 6 shows that the band gap of our $\mathrm{ZnO}$ electrodeposits increase with increasing carrier concentration. In the context of traditional donor/acceptor band theory, a decreased band gap would indicate an increased carrier concentration, as has been observed recently with Cl-doped $\mathrm{ZnO}$ electrodeposits. ${ }^{10}$ However, hydrogen doping in $\mathrm{ZnO}$ has been observed to go against this trend. ${ }^{5,6}$ Hydrogen-donated electrons can fill empty states in the conduction band of $\mathrm{ZnO}$ when doping levels are high enough to create a degenerate semiconductor, thereby leading to increased band gaps with higher doping levels (Moss-Burstein effect). ${ }^{4,8}$ Our data are consistent with this Moss-Burstein doping, whether for a single $p \mathrm{H}$ (Figure 6a for samples deposited at $p \mathrm{H} 6.5$ from a $\mathrm{Cl}^{-}$-free electrolyte) or over our whole range of deposition conditions (Figure 6b).

If we use the band gap $v s$. potential trend to attribute the increasing carrier concentration to higher $\mathrm{H}^{+}$doping levels, we arrive at an interesting - and rather counterintuitive - finding. Intuitively, one could reasonably expect that an increase in hydrogen generation would translate into more incorporated hydrogen and consequently a higher carrier concentration. However, we observe higher carrier concentrations at more positive deposition potentials, where there should be a lower rate of hydrogen generation. ${ }^{23}$ Thus, our findings suggest that the relationship between carrier concentration and the presence of hydrogen during the electrosynthesis process is more 
Shawn Chatman, Lisa Emberley, and Kristin M. PoduskaSignificant carrier concentration changes ...

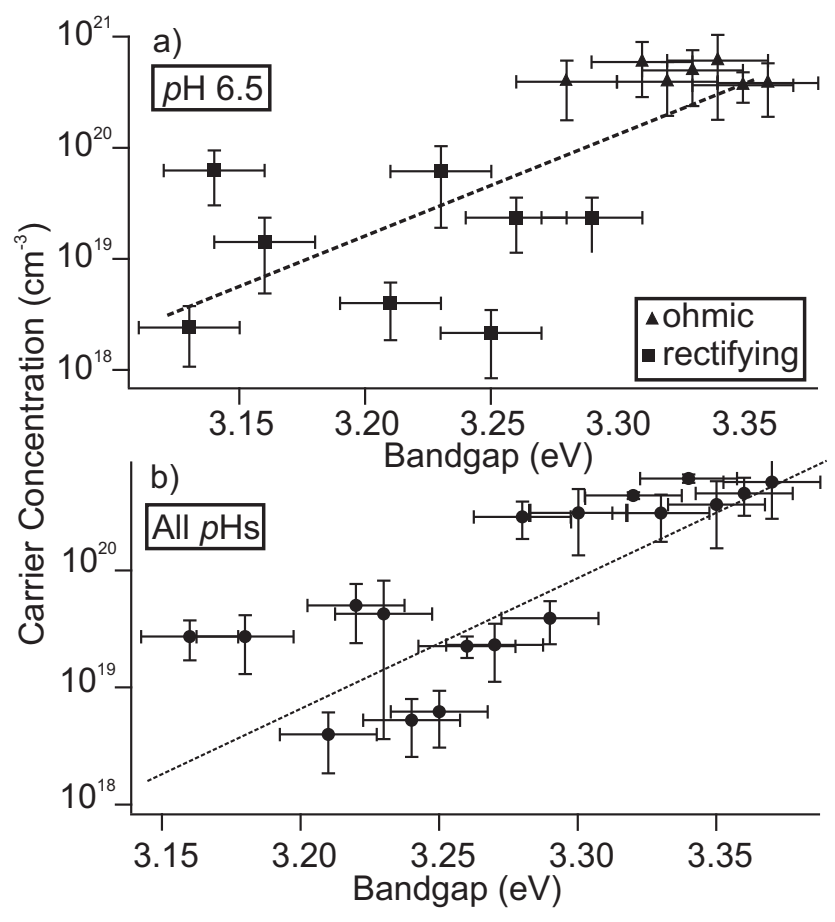

Figure 6: A plot of log carrier concentration versus band gap for deposition at $p \mathrm{H} 6.5$ (a) and all $p \mathrm{H}$ conditions (b). The line serves as a guide to the eye.

complicated.

One likely complicating factor is the presence of multiple types of defects. While recent studies indicate that hydrogen donors are an energetically favourable dopant, and thus the most dominant factor in the $n$-type doping in $\mathrm{ZnO}$, there is experimental evidence that supports the prevalence of oxygen and zinc vacancy, interstitial, and anti-site defect contributions. ${ }^{24}$ Of these intrinsic point defects, it is known that zinc vacancies, oxygen interstitials, and oxygen anti-sites act as deep acceptors within $\mathrm{ZnO}$ while oxygen vacancies, zinc interstitials, and zinc anti-sites act as donors. In fact, it has been found that in $\mathrm{ZnO}$, the formation of acceptor defects is energetically preferable to the formation of native donor defects. ${ }^{25,26}$ Zinc vacancy acceptors require the lowest formation energies of the three acceptor defects, and they are also stable to temperatures of $300^{\circ} \mathrm{C}$, which is far above our electrodeposition temperatures. Consequently, zinc vacancies would be the most likely kind of acceptor defect, according to thermodynamic arguments, and these acceptors could compensate for, and thus diminish the overall effect of, increased hydrogen incorporation. Previous studies have confirmed that the presence of deep acceptors in $\mathrm{ZnO}$ crystals will be active toward 
Shawn Chatman, Lisa Emberley, and Kristin M. PoduskaSignificant carrier concentration changes ...

hydrogen passivation in $\mathrm{ZnO}$. $^{7,27}$

If we adopt the working hypothesis that compensating defects could reduce the net $n$-type carrier concentration, it is reasonable to expect that these defects would be more prevalent at more negative deposition potentials. Reaction kinetics will be faster at greater overpotentials, and this increase in synthesis rate would likely contribute to an increase in defect densities. This is indeed consistent with the results shown in Figure 6.

Although $\mathrm{H}$ is now widely recognized as a prevalent dopant in $\mathrm{ZnO}$, it is still an open question where and how the $\mathrm{H}$ is incorporated. Theoretical studies ${ }^{7}$ have suggested locations for interstitial hydrogen, and data from infrared and Raman spectroscopic measurements ${ }^{28}$ have shown evidence of $\mathrm{H}$ incorporation. Computational investigations of $\mathrm{H}$ diffusion in $\mathrm{ZnO},{ }^{29}$ however, indicate that $\mathrm{H}$ complexation is likely based on the suprising thermal stability of incorporated $\mathrm{H}$ (up to 400 ${ }^{\circ} \mathrm{C}$ in some $\mathrm{ZnO}$ samples). In our samples, the resistance values and carrier concentration values are consistent over the span of months. Others have observed optical and electronic effects of $\mathrm{H}$ diffusion over the span of days to weeks. ${ }^{4,28}$ Because annealing can change not only $\mathrm{H}$ content but also surface structure, grain size, and oxygen content, it is not straightforward to ascribe annealing related changes in carrier concentration to changes in $\mathrm{H}$ content alone. Thus, pinpointing likely locations and chemical environments for $\mathrm{H}$ in the lattice is an ongoing challenge for all who work with $\mathrm{ZnO}$.

\section{Conclusions}

The large changes in carrier concentrations of native (unintentionally doped) electrodeposited $\mathrm{ZnO}$ are comparable to the effect of intentional extrinsic dopants like $\mathrm{Cl}^{-}$or borates. Since we observe that higher $n$-type carrier concentrations coincide with larger optical band gaps, we attribute the unintentional defects to the Moss-Burstein dopant hydrogen. Our data also indicate that both net carrier concentration and band gap are lower in samples deposited at more negative deposition potentials, contrary to the increase in hydrogen generation at more negative deposition potentials. 
Shawn Chatman, Lisa Emberley, and Kristin M. PoduskaSignificant carrier concentration changes ...

We attribute this trend to the higher deep acceptor defect density of samples deposited at more negative deposition potentials and their faster reaction kinetics.

There are inherent difficulties in quantitative assessments of hydrogen incorporation in semiconductors, particularly in assessing the hydrogen coordination environment, mobility, and complexation. For this reason, the study of donor and acceptor defects is receiving considerable attention from the scientific community, ${ }^{6,25}$ both theoretically and experimentally. The results of the present study highlight the need for better understanding of the interplay between native defects and external dopants.

\section{Acknowledgement}

We thank H. Gillespie (XRD) and L. Men (SEM) for use of instrument facilities at Memorial University of Newfoundland. We also acknowledge financial support from the Natural Science and Engineering Resource Council (Canada), Canada Foundation for Innovation New Opportunities Fund, and Memorial University of Newfoundland.

\section{Supporting Information Available}

Derivation of the Mott-Schottky capacitance relations and its relation to sample roughness, an explanation of the biased steel/ZnO/electrolyte junction characteristics, and a discussion of the effects of deposit thickness. This material is available free of charge via the Internet at http: //pubs.acs.org. 
Shawn Chatman, Lisa Emberley, and Kristin M. PoduskaSignificant carrier concentration changes ...

\section{References}

(1) Surek, T. J. Cryst. Growth 2005, 275, 292-304.

(2) Özgür, Ü.; Ya. I. Alivov,; Liu, C.; Teke, A.; Reshchikov, M. A.; Doğan, S.; Avrutin, V.; S.-J. Cho,; Morkoç, H. J. Appl. Phys. 2005, 98, 041301/1-103.

(3) Lincot, D. Thin Solid Films 2005, 487, 40-48.

(4) Ellmer, K. J. Phys. D: Appl. Phys. 2001, 34, 3097-3108.

(5) Van de Walle, C. G.; Neugebauer, J. Nature 2003, 423, 626-628.

(6) Chambers, S. A. Surf. Sci. 2007, 601, 5313-5314.

(7) Van de Walle, C. G. Phys. Rev. Lett. 2000, 85, 1012-1015.

(8) Burstein, E. Physical Review 1954, 93, 632-633.

(9) Ishizaki, H.; Imaisumi, M.; Matsuda, S.; Izaki, M.; Ito, T. Thin Solid Films 2002, 411, $65-68$.

(10) Rousset, J.; Saucedo, E.; Lincot, D. Chem. Mater. 2009, 21, 534-540.

(11) Izaki, M.; Omi, T. Appl. Phys. Lett. 1996, 68, 2439-2440.

(12) Ren, T.; Baker, H. R.; Poduska, K. M. Thin Solid Films 2007, 515, 7976-7983.

(13) Chatman, S.; Poduska, K. M. ACS Appl. Mat. Interfaces 2009, 1, 552-558.

(14) Yoshida, T.; Komatsu, D.; Shimokawa, N.; Minoura, H. Thin Solid Films 2004, 451-452, 166-169.

(15) Limmer, S. J.; Kulp, E. A.; Switzer, J. A. Langmuir 2006, 22, 10535-10539.

(16) Marotti, R.; Guerra, D.; Bello, C.; Machado, G.; Dalchiele, E. Sol.Energy Mater. Sol.Cells 2004, 82, 85-103. 
Shawn Chatman, Lisa Emberley, and Kristin M. PoduskaSignificant carrier concentration changes ...

(17) Mora-Seró, I.; Fabregat-Santiago, F.; Denier, B.; Bisquert, J. Appl. Phys. Lett. 2006, 89, 203117/1-3.

(18) Windsich, C. F.; Exarhos, J. J. Vac. Sci.Technol., A 1999, 18, 1677-1680.

(19) Fabregat-Santiago, F.; Garcia-Belmonte, G.; Bisquert, J.; Bogdanoff, P.; Zaban, A. J. Electrochem. Soc. 2003, 150, E293-E298.

(20) Pajkossy, T. Solid State Ionics 2005, 176, 1997-2003.

(21) Joint Commission on Powder Diffraction Standards - International Centre for Diffraction Data, "Powder Diffraction File", 2003.

(22) Peulon, S.; Lincot, D. J. Electrochem. Soc. 1998, 145, 864-874.

(23) Bard, A. J.; Faulkner, L. R. Electrochemical Methods : Fundamentals and Applications; John Wiley: New York, 2001.

(24) Vlasenko, L. S.; Watkins, G. D. Phys. Rev. B: Condens. Matter 2005, 72, 035203.

(25) Janotti, A.; Van de Walle, C. G. Phys. Rev. B: Condens. Matter 2007, 76, 165202/1-22.

(26) Oba, F.; Nishitani, S. R.; Isotani, S.; Adachi, H.; Tanaka, I. J. Appl. Phys. 2001, 80, 824828.

(27) Lavrov, E. V.; Weber, J.; Börrnert, F.; Van de Walle, C. G.; Helbig, R. Phys. Rev. B: Condens. Matter 2002, 66, 165205/1-7.

(28) Jokela, S. J.; McCluskey, M. D. Physica B 2007, 401-402, 395-398.

(29) Wardle, M. G.; Goss, J. P.; Briddon, P. R. Phys. Rev. Lett. 2006, 96, 205504/1-4. 


\section{Table of Contents graphic}

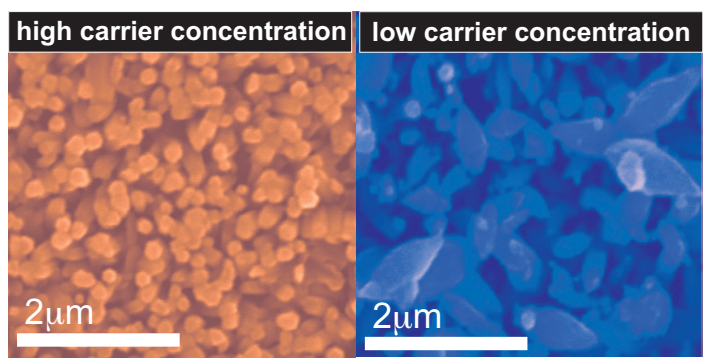

